\title{
Immersiveness of Ubiquitous Computing Environments Prototypes: A case study
}

\author{
Tiago Abade ${ }^{1,2}$, José C. Campos ${ }^{1,2}$, Rui Moreira ${ }^{1,2}$, Carlos C. L. Silva ${ }^{1,2}$, and \\ José Luís Silva ${ }^{3}$ \\ 1 Departamento de Informática, Universidade do Minho, Braga, Portugal \\ ${ }^{2}$ HASLab / INESC TEC, Braga, Portugal \\ ${ }^{3}$ Madeira-ITI/Universidade da Madeira, Funchal, Portugal \\ \{pg20691@alunos.uminho.pt, jose.campos@di.uminho.pt, \\ a49401@alunos.uminho.pt, carlos.l.silva@inesctec.pt, \\ jose.l.silva@m-iti.org\}
}

\begin{abstract}
The development of ubiquitous computing (ubicomp) environments raises several challenges in terms of their evaluation. Ubicomp virtual reality prototyping tools enable users to experience the system to be developed and are of great help to face those challenges, as they support developers in assessing the consequences of a design decision in the early phases of development. Given the situated nature of ubicomp environments, a particular issue to consider is the level of realism provided by the prototypes. This work presents a case study where two ubicomp prototypes, featuring different levels of immersion (desktop-based versus CAVE-based), were developed and compared. The goal was to determine the cost/benefits relation of both solutions, which provided better user experience results, and whether or not simpler solutions provide the same user experience results as more elaborate one.
\end{abstract}

Keywords: Ubiquitous computing, virtual environments, prototyping, evaluation, immersiveness, APEX

\section{Introduction}

In ubiquitous computing, a variety of computer devices are commonly used to assist and automate human tasks and activities in the physical world [13]. Due to the constant growth of information technologies, there has been a tendency to equip places of our daily life with ubiquitous computing devices. Currently, the main issue of developing such environments is that developers have to "rely on either low-fidelity techniques (such as paper prototypes and mental walkthroughs) or simply wait for a full scale deployment" [18] for testing and validation. This makes it inconvenient and costly to actually develop the systems. In many cases, it is not possible to install prototypes for testing purposes as that would be disruptive of ongoing activities. Hence, it is of utmost importance to find means of prototyping ubiquitous environments in a rapid fashion without disrupting key services of human society. 
Even though prototypes are not intended as final products, they must be both robust and refined for a fair evaluation [6]. The more detailed and realistic a prototype is, the more a user is able to discern the future utility of the prototyped system. The usefulness of an ubiquitous computing application will be determined by the developers' skills in identifying users' requirements and expectations. It is important that these requirements and expectations are met. By designing prototypes and letting users test them, a developer can better assess the potential impact and relevance that the system may have on a real scenario $[18,6]$.

The use of immersive Virtual Reality (VR) environments has been demonstrated to support accomplishing these goals [17]. We have created the APEX framework [17], that relies on a three-dimensional (3D) application server to create simulations for ubiquitous environments. This framework was sucessfuly used in the prototyping of ubiquitous computing environments, enabling the detection of potential user problems (functional [16] or non-functional [1]) in the early phases of development, while reducing the cost of redesign.

One of the goals of the work is that the framework must provide prototypes that feel real enough, allowing users to interact with the virtual environment in a way that feels natural. This can be accomplished through different levels of immersion, from simple desktops to more immersive and complex setups. For example, by taking advantage of the functionality of a CAVE (Cave Automatic Virtual Environment) [3], a virtual reality theater where a user can be immersed with stereoscopic 3D imaging.

On one hand, desktop-based prototypes provide a less immersive experience but are simpler and cheaper to develop and deploy. On the other hand, CAVEbased prototypes provide an more immersive user experience, but their are more complex to develop and interact with as well as more expensive to deploy. There is a need to compare and determine which solutions provide better user experience results and to which extent the added cost of a CAVE is justified. The goal of this paper is exactly to present one such comparison. The long term goal is to support developers evaluate the cost/benefits of each approach.

The paper is structured as follow: Section 2 presents related work. Section 3 describes the case study used (i.e. a smart library). The two user studies (Desktop-based versus CAVE-based) are presented in Section 4. Section 5 describes the results, and Section 6 discusses the results and summarizes with conclusions and future work.

\section{State of the Art}

Prototypes provide designers with a way of checking proposed solutions with low investment. However there is a tension between the quality of the results provided by the prototypes and the effort needed to make them close enough representations of the final systems once implemented. Prototypes should provide results which adequately reflect the experience of the physical implementation of the system on location. 
The prototyping of ubiquitous computing (ubicomp) systems can be approached from multiple perspectives, from prototypes of specific devices to prototypes of spaces equipped with such devices (see [4] for a good overview). We are specifically interested in the latter case.

Several approaches to the prototyping of ubicomp environments have been proposed $[9,19,11,17,10]$. Most of the approaches use virtual reality, some use Augmented Reality (e.g. the VARU framework [19]), and the work of Singh et al. [18] presents an initial approach to using immersive video. APEX [17] is unique in that, through a multi layer prototyping approach, it enables analysis at different levels of abstraction, from models of the system's behaviour to immersive virtual reality simulations.

Research results stated that User Experience (UX) evaluations can benefit from using virtual reality [14]. The veracity of evaluations in virtual environments (their ecological validity) has been addressed in many contexts. For example, Orland et al. [12] considered virtual worlds as representations of landscape realities and as tools for landscape planning suggesting their ecological validity. Scott [15] addresses it in the medical context and claims that virtual reality has promising ecological validity.

Solutions to improve different aspects of the immersion experience include, stereoscopic 3D, multi-display support and the use of external physical devices by providing a more natural style of interaction. How much immersion is enough and when simpler solutions provides the same user experience results has been discussed by Bowman and McMahan [2] in the general context of VR. A very recent work [5] compared three evaluation methods for mobile interactive systems: field-based studies, classical lab-based studies and evaluation using Immersive Video Environments (IVE). They claim that using IVE lead to identifying nearly the same number of major usability problems, similar effectiveness and task efficiency as the field-based study. The results suggest that in some areas IVE-based evaluations and field-based studies lead to similar results, while IVE exhibits most of the lab-based study benefits (e.g. repeatability and control). The authors present some initial insights into the benefits/drawbacks of the three evaluation methods, but state that further research is needed to fully understand which method is more appropriate for a particular situation.

While the evaluation using IVE seems to be an adequate method for some areas, VR simulations seem a more promising approach in the case of ubicomp environments.

\section{A smart library prototype}

In order to compare the experience of using APEX-based prototypes at different levels of immersion, an exiting prototype of a smart library was used (see [1]). The user visible component of the prototype consists of a virtual world developed in OpenSimulator ${ }^{4}$ (an open source multi-platform, multi-user 3D application server) and accessed through an appropriate viewer.

\footnotetext{
${ }^{4}$ http://opensimulator.org (last accessed February 20, 2015).
} 


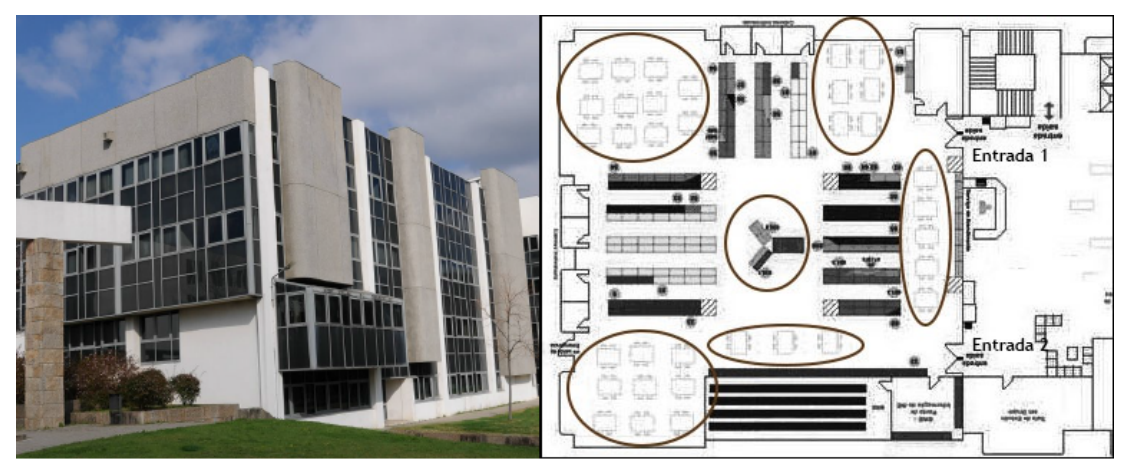

Fig. 1. a - Library of the University of Minho; b - Table sections of the library

The physical library on which the prototype is based, is located in the Gualtar campus of the Minho University in Braga, Portugal (see Figure 1a). The building has 3 floors, including a ground-floor where the main reception is located. The other two floors are identical and have reading and study rooms (see Figure 1b). Each of these two floors is composed of a reception (on the right in the figure) flanked by doors that give access to the reading/study rooms. These rooms have 6 table sections (marked with circles in Figure 1b).

The prototype used represented the enhancement of the library with ubicomp technology providing indication of seat availability within each floor. Two types of information panels were simulated to provide information about seats in the library. The first type (the alphanumeric panel - see Figure 2c) shows information on seat availability in a textual format. Two alphanumeric panels were placed on every floor, at the main entrance and in the center of every reading/studying room. The second type of information panel presents a depiction of the floor plan of the library, and indicates the availability of seats using red and green LEDs lights (see Figure 2d). LED panels were located at the entrance of each floor.

Virtual presence sensors were placed in the environment. Every time a sensor is triggered, this information is sent to every screen present in the building to refresh the data displayed.

\section{User studies: Desktop versus Cave}

The prototype described in the previous section was evaluated through two user studies. More than evaluating the actual system being prototyped, the main focus of the studies was in understanding whether the prototype could be useful in performing such evaluation. And, in particular, to compare different levels of immersion in the deployment of the prototypes. Hence the users studies used different deployment setups. In one case the prototype was presented to users on a desktop machine, to create what we consider a low immersion condition. In 

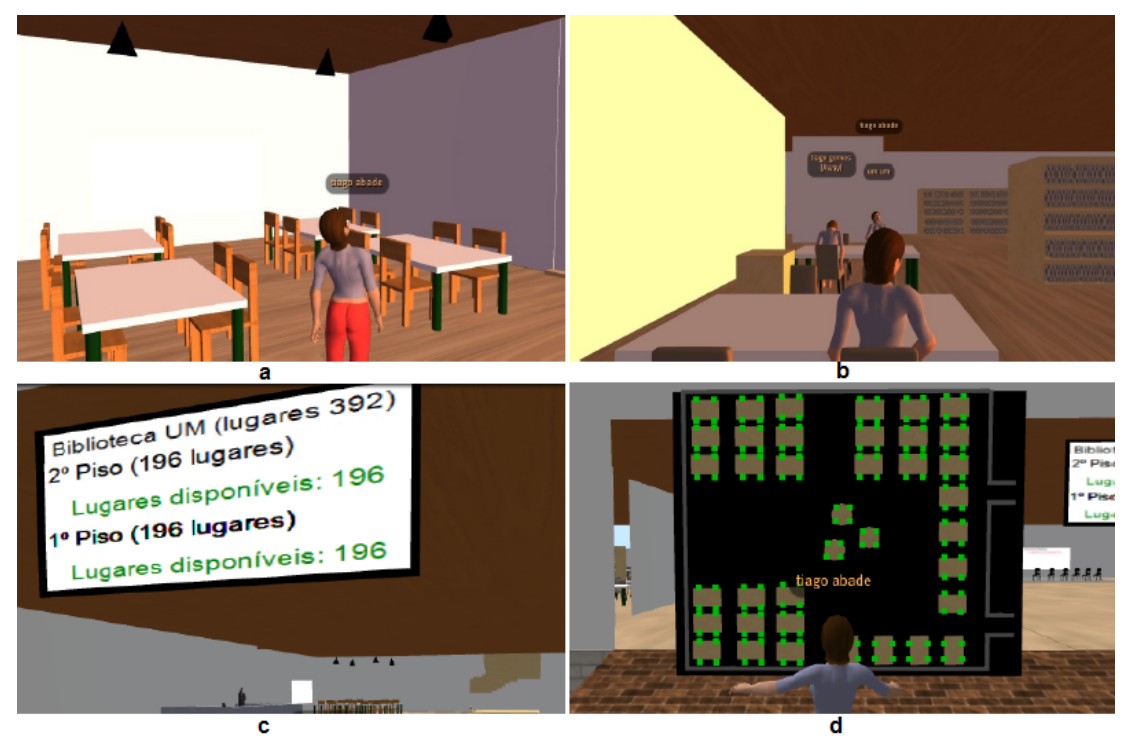

Fig. 2. OpenSimulator representation of the Library: a - ceiling movement sensors (highlighted with triangular markers); b - sitting at a table triggers pressure sensors ; c - alphanumeric panel; d - LED panel.

the other case, the high immersion condition, the prototype was deployed in a CAVE environment, using stereoscopic projection.

\subsection{Low immersion setup}

In the low immersion condition the prototype was presented to users on a 22 " Samsung SyncMaster 3D 2233RZ screen. In effect, the machine used to run APEX (a Intel Core i5-2400 machine with two Asus GeForce GTX460 1GB DirectCU 2DI GDDR5 PCI-E GPUs) featured 3 such screens, but for this setup only one screen was being used to present the virtual world (see Figure 3 ).

Interaction with the environment was achieved, in typical fashion, via keyboard and mouse. The viewer used was the CoolVLViewer.

\subsection{High immersion setup}

In the high immersion condition, a CAVE available at the Laboratory of Perception and Vision from CCG (The Computer Graphics Center) in Guimarães, Portugal was used. The CAVE in question was composed of three rear projection screens set as a single flat screen, which means that they were lined up on a single plane right next to each other. As represented in Figure 4, the system 


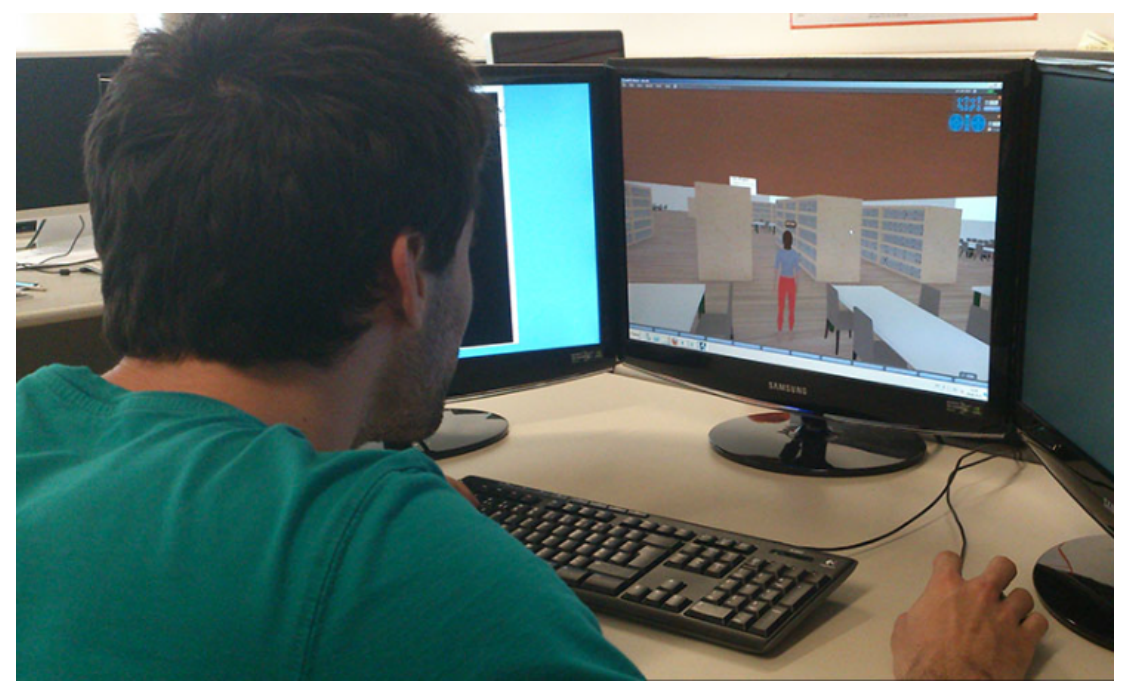

Fig. 3. Low immersion setup

is a cluster with three machines (lvp-node0, lvp-node1 and lvp-node2), one for each projector.

The CAVE had support for NVIDIA 3D Vision technology. Each machine of the cluster had a NVIDIA Quadro 4500 graphics card. The projectors (Christie Mirage S+4k) had active stereo support. Deploying APEX on the CAVE, however, presented some problems. The first was identifying a viewer able to produce a stereoscopic view of the virtual world. The second, projecting views of the world through the three projectors in the CAVE. Remember that typical viewers for OpenSimulator are designed for a desktop use on a single screen.

In [7] an analysis of the alternatives was carried out. In short, the conclusion was that, while it was possible to have a stereoscopic view of the virtual world (through the Dale's SL Viewer) and to have three (or more) coordinated views of the world projected in different screens, with currently available viewers having three coordinated stereoscopic views was not feasible. The Dale's SL Viewer can only be used to generate one view into the world. Because each machine is directly connected to one projector only, we were restricted to using a single projector. Although a single projector setup did not actually surround a user in a cubic fashion, it does provide a larger image that results in a more immersive experience than obtained with the desktop screen. Additionally, the use of 3D enhanced the experience.

Testing Dale's SL Viewer's stereoscopic 3D modes, inside the CAVE, resulted in the following:

- Anaglyph stereo: since this mode is compatible with almost any kind of system, no problems happened when using it. With the appropriate 3D red/cyan 


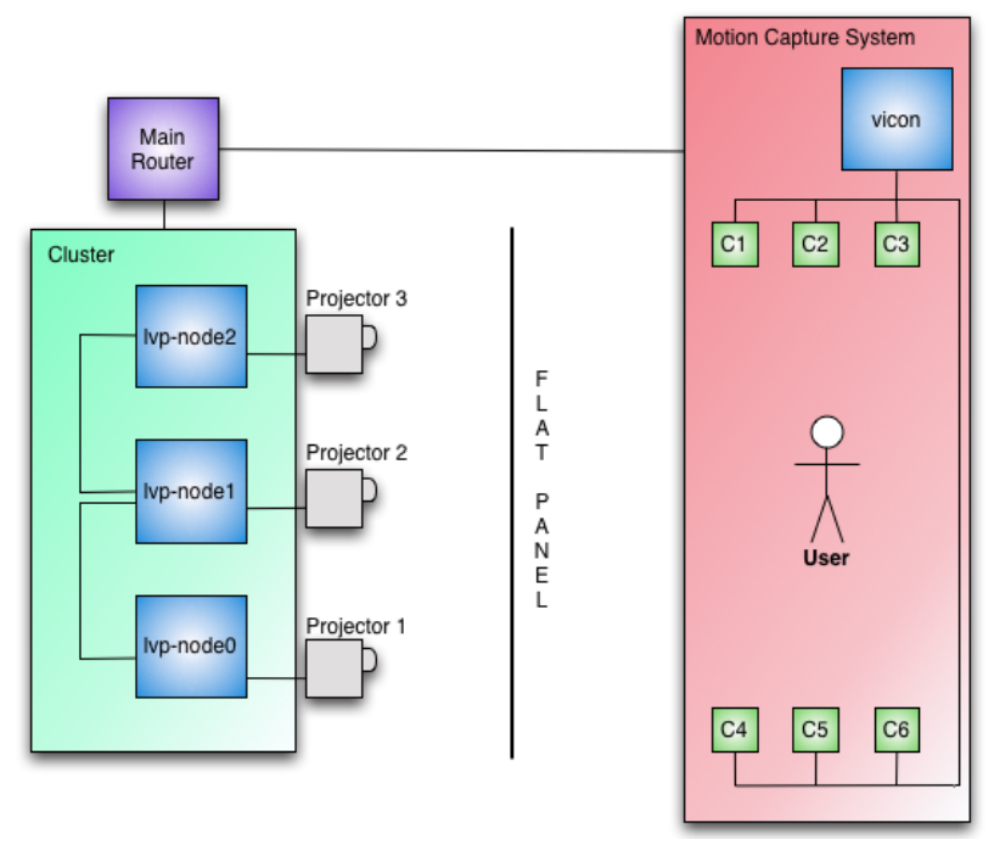

Fig. 4. CAVE in the LVP

glasses, the user can see a three-dimensional scene as a result of the binocular disparity depth cue provided by the anaglyph. Figure 5 shows Dale's SL Viewer running in anaglyph stereo mode inside the CAVE;

- Active stereo: taking into account the hardware used for the CAVE, i.e. the graphics cards have 3-pin din output that can be used to connect to the projectors via GPIO, and the NVIDIA Quadro graphics cards have OpenGL quad-buffered stereo support for NVIDIA 3D Vision, it is possible to obtain stereoscopic 3D using this mode. However, testing did not show adequate results. This may be due to abnormal configuration of the projectors and graphics drivers;

- Passive stereo: considering that the projectors do not have polarized filters, this mode was not supported.

Given the above, anaglyph stereo was used in the user tests. Note that since running the user studies, the CAVE has suffered and extensive overhaul and the use of Active stereo is now feasible.

Interaction was achieved through the use of a Wiimote controller, programmed to act as and input device to the virtual world. Test subjects could wither use the Wiimote's movement or keys to control the avatar representing them in the virtual world. 


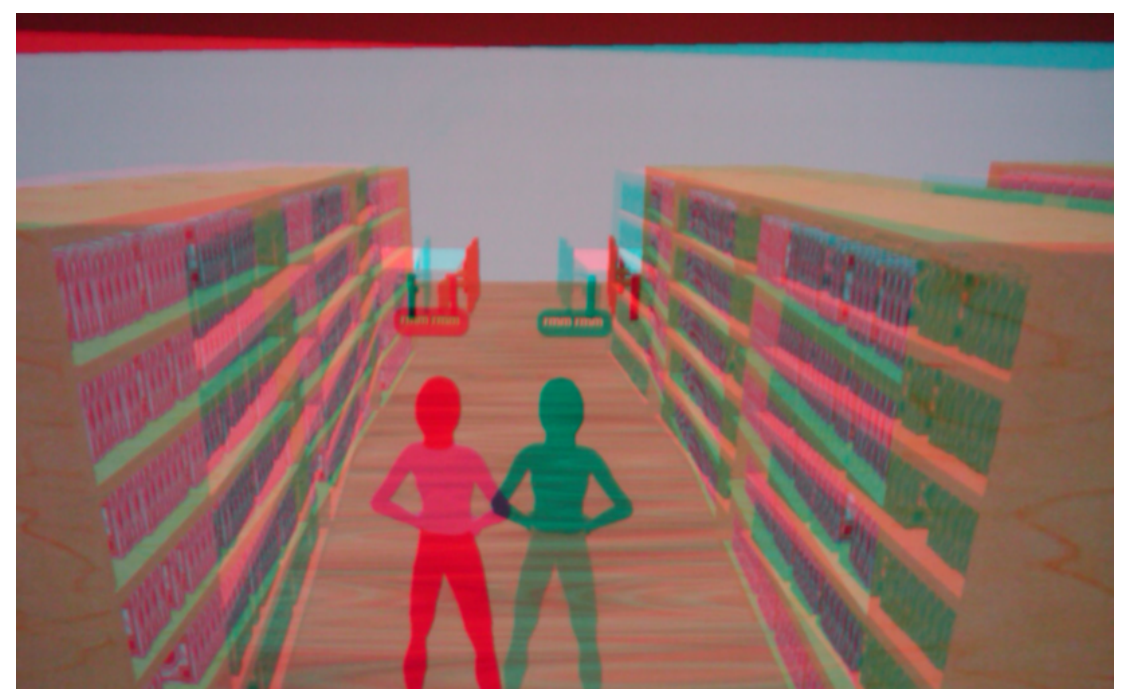

Fig. 5. Anaglyth stereo in the CAVE

\subsection{Study setup}

As described earlier one goal of the study was to assess whether significant differences could be found in the experience of using the prototype under different immersion conditions. Considering that students are the largest group of users of the library, test subjects were recruited from the student population. In total 21 students participated in the study. A between subjects setup was used with 12 students integrating the low immersion condition and 9 integrating the high immersion condition.

The students were briefed with a short introduction about the purpose of the prototype and the information they would find in the simulation. During this phase they had also the opportunity of exploring the environment in the relevant immersion condition.

After that initial phase, they were asked to enter the library and find an available seat, using the screen panels' assistance if they so wished. Each student used the simulation individually but bots were introduced in several seats to improve the realism of the experience. Students were free to explore the environment without being disturbed. No time restrictions were placed in relation to accomplishing the designated task of finding a place to seat.

While in the virtual environment students were observed, their movements and the steps they took to find a place to sit noted. After completing the tasks, students were asked to answer a questionnaire. Besides related to the characterization of the test subjects, the questionnaire was composed of 30 questions, addressing topics related to the screen panels and to the use of the virtual en- 


\begin{tabular}{|c|c|c|c|c|c|c|c|c|c|c|c|}
\hline & & & ov & $\mathrm{mr}$ & ersi & & & igh & $\mathrm{mr}$ & $\mathrm{rsi}$ & \\
\hline & & 1 & 2 & 3 & 4 & 5 & 1 & 2 & 3 & 4 & 5 \\
\hline 1 & Able to recognize the environment & & & 1 & 9 & 2 & & & 2 & 7 & \\
\hline 2 & Helps analysis of alternatives & & & 1 & 3 & 8 & 1 & & 2 & 5 & 1 \\
\hline 3 & Advantageous to create prototypes & & & & 2 & 10 & & & & 3 & 6 \\
\hline 4 & Advantageous to introduce panels & & & & 1 & 11 & & & & 5 & 4 \\
\hline 5 & Panels are well located & & 3 & 5 & 4 & & 1 & 1 & 4 & 2 & 1 \\
\hline 6 & Would only use LED panels & & 5 & 4 & & 3 & 1 & 3 & & 3 & 2 \\
\hline 7 & I would recommend the approach & & & & 2 & 10 & & & 1 & 5 & 3 \\
\hline 8 & I would use the approach & & & & 4 & 8 & & & & 4 & 5 \\
\hline
\end{tabular}

Table 1. Comparison of some results

vironment as a prototype. Answers were given in a 5 points Likert scale. The questionnaire was divided into the following sections:

- Profile of the students, including their experience of games using controls similar to those used in this user study.

- Evaluation of the prototype as a tool for evaluation. Here, aspects such as immersion, utility and user satisfaction were measured.

- Evaluation of the prototyped solution, including questions concerned with the location, design and behaviour of the screen panels.

Twelve students, with an average age of 23 years, participated in the low immersion condition of the study. Of the 12 students that participated in the study, 10 declared they were familiar with third-person gaming.

Nine test subjects, with an average age of 28 years, participated in the high immersion condition of the study. Of the 9 students, 5 declared they were familiar with third-person gaming.

\section{$5 \quad$ Results}

The two main aspects addressed in the study will now be discussed. To simplify presentation, only the more relevant/illustrative questions are addressed.

Table 1 presents a summary of the result discussed below. For each of the questions, the number of times a particular value in the Likert scale was chosen is indicated. Modes are highlighted in boldface. We run a Mann-Whitney test for differences across two independent samples, and we found significant differences across groups (low vs high immersion) in three of the items in Table 1:

- Question 2: The approach helps analysis of alternatives $(\mathrm{p}=0,013)$

- Question 4: The introduction of panels would be advantageous $(\mathrm{p}=0,021)$ 
- Question 7: I would recommend the approach $(\mathrm{p}=0.020)$

In all cases the low immersion condition had an higher score than the high immersion condition. However, looking at Table 1 responses to these three questions all have a mode on the positive side of the scale (totally agree vs. agree).

\subsection{The prototype as an evaluation artifact}

In both cases test subjects were able to identify the environment (mode of 4). Results in the first condition were slightly more positive (answers were in the range 3 to 5 ; against 3 to 4 in the second condition) but that can be attributable to the test subjects in the first study being more familiarized with the library as the test subjects in the second study were on a different campus of the University.

Also in both cases there was agreement that the approach helps in the design process. For example the question regarding support for identifying alternative solutions had a mode of 5 in the first condition and 4 in the second. Interestingly the answers were more positive in the low immersion scenario, when compared to the high immersion scenario. We posit that this might be due to the more 'intimidating' setup of the CAVE, but this is something that needs further research. In both conditions there was a mode of 5 in the answer to the question regarding whether the subjects saw an advantage in building a virtual reality representation of the system before actual physical deployment. Similarly, the answers to the questions regarding whether the test subjects would use or recommend the approach all had positive results, with a mode of five in all cases, except for the question about recommending the approach, which in the high fidelity condition had a mode of 4 . Again, we believe that the fact that a CAVE was used might have made the test subjects less confident that it would be feasible to use the approach on a regular basis but more research is needed in this case.

\subsection{Screen panels' evaluation}

Regarding consistency of the results, it can be observed that in both conditions there was agreement that the introduction of the panels in the library would be beneficial. The mode was 5 in the first condition and 4 in the second, but answers in that second case were almost equally distributed between 4 and 5 ( 5 votes against 4). A mode of 3 was obtained in both cases in the question regarding whether the panels' location was adequate.

Regarding the question of whether using the LED panels only would be enough, the answer was negative in the low immersion condition (mode of 2), while the observation of the test subjects' behavior showed that they resorted mainly to these panels. Hence there was some contradiction between observed behavior and the results of the questionnaire. In the high immersion condition this discrepancy was less obvious as the result was bi-modal (split between disagree and agree). However, again in this case observation of the test subjects showed a clear preference for the LED panels. Determining whether this is representative 
of actual usage will require physical installation of the system. However, in previous work we were able to observe that the user's behavior in the virtual world was consistent with their behavior in the physical world [8].

\section{Discussion and Conclusions}

In this paper we have addressed the issue of how immersive ubicomp prototypes need to be. A virtual reality based prototype of a ubicomp system was tested on both a desktop computer and in a CAVE. Overall there was no major significant differences in the results of the two conditions. This indicates that for the particular needs of the prototyping approach used, the added cost of deploying the prototypes in a CAVE environment does not seem to pay off.

Whether the results of the tests adequately reflect the behavior and experience of users in the actual system once fielded was not the issue in this case. In previous work we have addressed this issue [8], and the ecological validity of virtual worlds has also been argued for in the literature $[15,12]$.

A limiting factor of the study that was carried out is the fact that we were not able to use a fully immersive setup in the CAVE. The setup used did represent, at the time, the best that could be achieved with the APEX framework in the available CAVE. In this sense the study faithfully represents the available alternatives. Since then, the CAVE has been subject to a major overhaul and a more immersive setup is now possible. We plan to repeat the study in this setup, and also add, as an intermediate condition, a 3D desktop setup.

\section{Acknowledgments}

This work is funded by Fundação para a Ciência e a Tecnologia (FCT) through Projecto Estratégico - LA 9 - 2014-2015 (PEst-OE/EEI/LA0009/2015).

\section{References}

1. Tiago Abade, Tiago Gomes, José Luís Silva, and José C. Campos. Design and evaluation of a smart library using the apex framework. In Norbert Streitz and Panos Markopoulos, editors, Distributed, Ambient, and Pervasive Interactions, volume 8530 of Lecture Notes in Computer Science, pages 307-318. Springer, 2014.

2. Doug A Bowman and Ryan P McMahan. Virtual reality: how much immersion is enough? Computer, 40(7):36-43, 2007.

3. Carolina Cruz-Neira, Daniel J. Sandin, Thomas A. DeFanti, Robert V. Kenyon, and John C. Hart. The CAVE: Audio visual experience automatic virtual environment. Communications of the ACM, 35(6):64-72, June 1992.

4. Nigel Davies, James Landay, Scott Hudson, and Albrecht Schmidt. Guest editors' introduction: Rapid prototyping for ubiquitous computing. IEEE Pervasive Computing, 4(4):15-17, 2005. 
5. Ioannis Delikostidis, Holger Fritze, Thore Fechner, and Christian Kray. Bridging the gap between field- and lab-based user studies for location-based services. In Georg Gartner and Haosheng Huang, editors, Progress in Location-Based Services 2014, Lecture Notes in Geoinformation and Cartography, pages 257-271. Springer, 2015.

6. Steve Hodges, Shahram Izadi, and Simon Han. wasp: a platform for prototyping ubiquitous computing devices. Proceedings of the 1st International Workshop on Software Engineering Challenges for Ubiquitous Computing (SEUC 2006), 2006.

7. Rui Moreira. Integrating a 3D application server with a CAVE. MSc thesis, University of Minho, 2011.

8. Samuel Moreira. Simulating Ubiquitous Computing Environments. MSc thesis, University of Minho, 2013.

9. Ali A Nazari Shirehjini and Felix Klar. 3dsim: rapid prototyping ambient intelligence. In Proceedings of the 2005 joint conference on Smart objects and ambient intelligence: innovative context-aware services: usages and technologies, pages 303307. ACM, 2005.

10. Yoosoo Oh, Changgu Kang, and Woontack Woo. U-vr simulator linking real and virtual environments based on context-awareness. In Proc. International Workshop on Ubiquitous Virtual Reality (ISUVR 2009), pages 052-055, 2009.

11. Eleanor O'Neill, David Lewis, and Owen Conlan. A simulation-based approach to highly iterative prototyping of ubiquitous computing systems. In Proceedings of the 2nd International Conference on Simulation Tools and Techniques, page 56. ICST (Institute for Computer Sciences, Social-Informatics and Telecommunications Engineering), 2009.

12. Brian Orland, Kanjanee Budthimedhee, and Jori Uusitalo. Considering virtual worlds as representations of landscape realities and as tools for landscape planning. Landscape and urban planning, 54(1):139-148, 2001.

13. Stefan Poslad. Ubiquitous computing: smart devices, environments and interactions. 2009

14. Francisco Rebelo, Paulo Noriega, Emília Duarte, and Marcelo Soares. Using virtual reality to assess user experience. Human Factors: The Journal of the Human Factors and Ergonomics Society, pages 964-82, 2012.

15. Fiona Scott. An Investigation into the Ecological Validity of Virtual Reality Measures of Planning and Prospective Memory in Adults with Acquired Brain Injury and Clinical Research Portfolio. PhD thesis, University of Glasgow, 2011.

16. José Luís Silva, José C. Campos, and Michael D. Harrison. Formal analysis of ubiquitous computing environments through the APEX framework. In Symposium on Engineering interactive computing systems, pages 131-140. ACM SIGCHI, 2012.

17. José Luís Silva, José C. Campos, and Michael D. Harrison. Prototyping and analysing ubiquitous computing environments using multiple layers. International Journal of Human-Computer Studies, 72(5):488 - 506, 2014.

18. Pushpendra Singh, Hai Nam Ha, Patrick Olivier, Christian Kray, Zhiwen Kuang, Amy Weihong Guo, Phil Blythe, and Phil James. Rapid prototyping and evaluation of intelligent environments using immersive video. In 8th International Conference on Human Computer Interaction with Mobile Devices and Services (Mobile HCI 2016), pages 264-264. ACM, 2006.

19. Lode Vanacken, Joan De Boeck, Chris Raymaekers, and Karin Coninx. Designing context-aware multimodal virtual environments. In Proceedings of the 10th international conference on Multimodal interfaces, pages 129-136. ACM, 2008. 